\title{
Factores demográficos y psicosociales asociados al exceso de peso en una población de Colombia
}

\author{
Demographic and lifestyle factors associated to weight excess in a \\ Colombian population
}

\author{
Buitrago-Gómez $M A^{1}$, Uribe-Forero $M C^{1}$, Cabarique-Pardo $L R^{1}$, Marín-Carrillo $L F^{2}$, \\ Serrano-Gómez $S E^{1}$, Wandurraga $E A^{1,3}$.
}

${ }^{1}$ Universidad Autónoma de Bucaramanga (UNAB).

${ }^{2}$ Centro Médico Carlos Ardilla Lulle, Floridablanca, Colombia.

${ }^{3}$ Endocrinólogos del Oriente, FOSCAL Internacional,

Floridablanca, Colombia.

Autor de correspondencia: Mario Alejandro Buitrago-Gómez

Correo electrónico: mbuitrago701@unab.edu.co

Fecha de recepción: 9/03/2020

Fecha de aceptación: 1/12/2020

\section{Resumen}

Introducción: la obesidad es una enfermedad de alto interés en salud pública y de etiología multifactorial. Es necesario conocer los aspectos ambientales propios de cada región que contribuyen al desarrollo de esta patología. El objetivo del estudio fue evaluar en la actualidad en una población colombiana la asociación entre diversos factores demográficos y psicosociales de la infancia y la edad adulta con tener exceso de peso.

Materiales y métodos: se realizó un estudio observacional, descriptivo y de corte transversal mediante una encuesta electrónica realizada a pacientes y acompañantes en la Clínica FOSCAL, donde se evaluaron las variables demográficas, las condiciones de vida en la infancia y los hábitos actuales. Fueron incluidos sujetos mayores de 18 años. Las personas con déficit cognitivo, pérdida involuntaria de más del $10 \%$ del peso y embarazadas fueron excluidas.

Resultados: en el análisis se incluyeron 490 participantes con edad promedio de 31,4 \pm 15 años; el 58,8 \% fue mujeres y el 91,4 \% procedía del área urbana. El 44,8 \% de los encuestados tenía exceso de peso (32,6 \% sobrepeso y 12,2\% obesidad). Se encontró asociación entre la edad actual (OR: 1,11; IC $95 \%$ : 1,04-1,19; $p=0,0,002$ ), el peso alcanzado a los 18 años (OR: 1,10; IC 95\%: 1,06-1,15; $p<0,001$ ) y haber vivido en la infancia en un conjunto residencial (OR: 0,40; IC 95 \%: 0,18-0,88; $p<0,001)$ con tener exceso de peso en la actualidad. Se apreció una ganancia de peso de $0,35 \mathrm{~kg}$ por año de vida cumplido. Otros hallazgos, como el consumo de alcohol y sustancias psicoactivas, así como la actividad física semanal no tuvieron asociación.

Conclusiones: la edad, el peso alcanzado a los 18 años y el no haber vivido en un conjunto residencial en la infancia fueron los factores asociados a tener exceso de peso en una población adulta colombiana.

Palabras clave: etiología, sobrepeso, factores sociológicos, índice de masa corporal, obesidad, sobrepeso.

\section{Abstract}

Introduction: Obesity is a disease of high interest in public health of multifactorial etiology. It is necessary to know the own environmental aspects of each region that contribute to the development to this disease. The aim was to evaluate the association between diverse demographic and psychosocial factors in childhood and adulthood with having weight excess in the present in a Colombian population.

Materials and methods: We conducted a descriptive cross-sectional study. An electronic poll that evaluated demographic variables, childhood conditions and actual habits was applied to subjects older than 18 years who were attending to FOSCAL Hospital as patients or companions. We excluded people with cognitive deficiency, involuntary weight loss of $10 \%$ or more and pregnant women.

Results: 490 participants were included in the analysis with a mean age of $31.4 \pm 15$ years; $58.8 \%$ were women and $91.4 \%$ came from urban area. $44.8 \%$ of the participants had weight excess $(32.6 \%$ had overweight and $12.2 \%$ had obesity). We found association between actual age (OR: 1.11; CI $95 \%: 1.04-1.19 ; p=0.0 .002)$, weight achieved at the age of 18 (OR: 1.10; CI $95 \%: 1.06-1.15 ; p<0.001)$ and have lived in a residential unit during childhood (OR: 0.40; CI $95 \%$ : 0.18-0.88; 
$p<0.001)$ with having weight excess in the present. A weight gain of $0.35 \mathrm{~kg}$ per year of life was documented. Other findings such as alcohol and psychoactive substances as well as weekly performed physical activity, were not associated.

Conclusions: Actual age, weight achieved at the age of 18 and have not lived in a residential unit were the associated factors to have weight excess in an adult Colombian population.

Keywords: Etiology; overweight; Sociological factors; Body mass index; Obesity; Overweight.

\section{Introducción}

La obesidad hace parte de las enfermedades crónicas no transmisibles, considerada por la Organización Mundial de la Salud (OMS) como una prioridad en salud pública debido a su alta prevalencia y carga de morbilidad. Se estima que alrededor del 27,5 \% de la población mundial tiene exceso de peso (entendido como sobrepeso u obesidad) (1). En el 2015, la Encuesta Nacional de Salud estimó una prevalencia de exceso de peso del 56,5 \% en la población colombiana adulta (sobrepeso, $37,8 \%$ y obesidad, $18,7 \%$ ) (2).

Desde el punto de vista fisiopatológico, la obesidad consiste en la acumulación excesiva de grasa en el tejido adiposo (3), que ocasiona morbilidad metabólica y mecánica. Su evaluación clínica se determina con el índice de masa corporal (IMC), el cual establece que una persona tiene sobrepeso u obesidad cuando es superior a $25 \mathrm{~kg} / \mathrm{m}^{2}$ y $30 \mathrm{~kg} / \mathrm{m}^{2}$, respectivamente (4). El uso de esta herramienta ha sido aceptado por la OMS como método diagnóstico, en conjunto con la medición del perímetro de la cintura y la presencia de comorbilidades (5).

El desarrollo de la obesidad comprende tanto factores modificables (dieta con una alta densidad energética, sedentarismo, bajo estrato socioeconómico y nivel educativo, entre otros) $(6,7)$, como no modificables, los cuales incluyen edad, sexo femenino y espectro de heredabilidad genética de la obesidad, que abarca desde la obesidad monogénica y sindrómica hasta la obesidad común (8).

Así mismo, el exceso de peso se ha descrito como factor de riesgo en un amplio espectro de patologías como la enfermedad cardiovascular (infarto agudo de miocardio, accidente cerebrovascular e insuficiencia cardíaca) (9), enfermedades metabólicas relacionadas con la resistencia a la insulina (diabetes mellitus, síndrome metabólico, síndrome de ovario poliquístico e hígado graso) (10-13) y mecánicas, como la apnea obstructiva del sueño y la osteoartritis $(14,15)$. Adicionalmente existe una asociación con una mayor incidencia de neoplasias, como el cáncer de endometrio y colorrectal $(16,17)$.

Teniendo en cuenta que la etiopatogenia de la obesidad es multifactorial y que comprende factores genéticos y ambientales, es necesario investigar sobre los posibles aspectos que contribuyen al desarrollo de esta en cada cultura o región (18, 19). El objetivo de esta investigación fue evaluar la asociación entre diversos factores demográficos y psicosociales con tener exceso de peso en una población adulta.

\section{Materiales y métodos}

Se realizó un estudio observacional descriptivo de corte transversal con componente analítico, mediante la aplicación de una encuesta desarrollada por el equipo investigador, en un formato de Google Forms (Anexo 1). El instrumento fue aplicado de forma presencial por encuestadores previamente capacitados y por vía digital mediante invitación electrónica, donde a través de una invitación pública en medios de difusión digitales, como correo electrónico o redes sociales, se accedía a un formato de diligenciamiento en línea. Se incluyeron hombres y mujeres mayores de 18 años que se encontraran en calidad de pacientes o acompañantes en las diferentes áreas del complejo FOSCAL y FOSCAL Internacional (en sala de espera de ENDORIENTE, Higuera Escalante, radiólogos especializados o en cafetería), o que voluntariamente abrieran el link de acceso público a la encuesta por vía digital. En ambas modalidades se solicitó consentimiento informado electrónico como requisito para el diligenciamiento de la encuesta, dando por entendido el consentimiento cuando posterior a su lectura, haciendo énfasis en el carácter voluntario de su participación y anonimato, el potencial participante oprimía el botón "aceptar" y contestaba las preguntas. Las personas con alteraciones cognitivas o para la comunicación, historia de cirugía bariátrica, enfermedad crónica con pérdida de más del $10 \%$ del peso y mujeres embarazadas fueron excluidas.

El instrumento empleado comprendió 65 preguntas y evaluó variables sociodemográficas (edad, sexo, procedencia, estrato socioeconómico actual, nivel educativo alcanzado y número de hermanos), variables relacionadas con la infancia (edad gestacional, peso al nacer, lactancia materna, haber pertenecido a una selección deportiva, alimentación en el colegio, estrato socioeconómico en la infancia, tipo de vivienda, peso a los 18 años, matoneo y maltrato por los padres) y variables relacionadas con estilos de vida actuales (actividad física en la ocupación, práctica deportiva, actividad física de los padres, tabaquismo, alcohol o consumo de drogas ilícitas en algún momento de la vida y puntaje IPAQ [international physical activity questionnare]).

El muestreo fue por conveniencia y se buscó diligenciar 500 encuestas. El protocolo fue aprobado por el comité de ética de la FOSCAL. Posterior a la inclusión de los participantes en el estudio y recolección de los datos, se realizó una depuración de la base de datos, excluyendo datos extremos o no plausibles biológicamente. Se realizó un análisis descriptivo univariado mediante medidas de tendencia central y dispersión de las variables cuantitativas, promedio y desviación estándar, frecuencia relativa y absoluta para las variables cualitativas. Por último, se realizó un análisis multivariado mediante el método de selección intencionada de covariables backward, donde se 
trabajó con un alpha de 0,05. Para el análisis y depuración de datos se utilizó el programa STATA versión 15.

\section{Resultados}

Se diligenciaron 500 encuestas y se excluyeron 10 por información incompleta. Un total de 490 sujetos fue incluido en el análisis, de los cuales, 288 (58,7 \%) fueron mujeres, y el promedio de edad fue de 31,4 15 años. El 78,7 \% de la población entrevistada era de Santander y el 91,4 \% procedía de zona urbana. Un total de 290 personas (59,1 \%) tenía educación superior.

Respecto a la antropometría, el promedio del peso fue de $70 \pm 14,7 \mathrm{~kg}$, con un IMC de $25 \pm 14,6 \mathrm{~kg} / \mathrm{m}^{2}$. El 44,8\% tenían exceso de peso, lo que correspondía en 160 sujetos (32,6\%) a sobrepeso y en $60(12,2 \%)$ a obesidad. En relación con el peso logrado a los 18 años, el promedio fue de $61,4 \pm 12,2 \mathrm{~kg}$.

Las variables categóricas demográficas y psicosociales en relación con tener exceso de peso se muestran en las Tablas 1 y 2 . Se encontró asociación entre la edad actual y tener exceso de peso, con un promedio de edad de 26,6 años para normopeso y de 37,1 años para aquellos que tenían exceso de peso $(p<0,001)$. En la Figura 1 se muestra el delta entre el peso actual y el logrado a los 18 años. De igual manera, se encontró asociación positiva con el número de hermanos $(p<0,001)$. En contraste, no existió correlación entre el exceso de peso con el número de meses de lactancia materna o el número de cigarrillos promedio/día.

Figura 1. Delta entre el peso actual y el peso a los 18 años (490 sujetos entrevistados $>18$ años). Por cada año cumplido se ganaron $0,35 \mathrm{~kg}^{*}$.

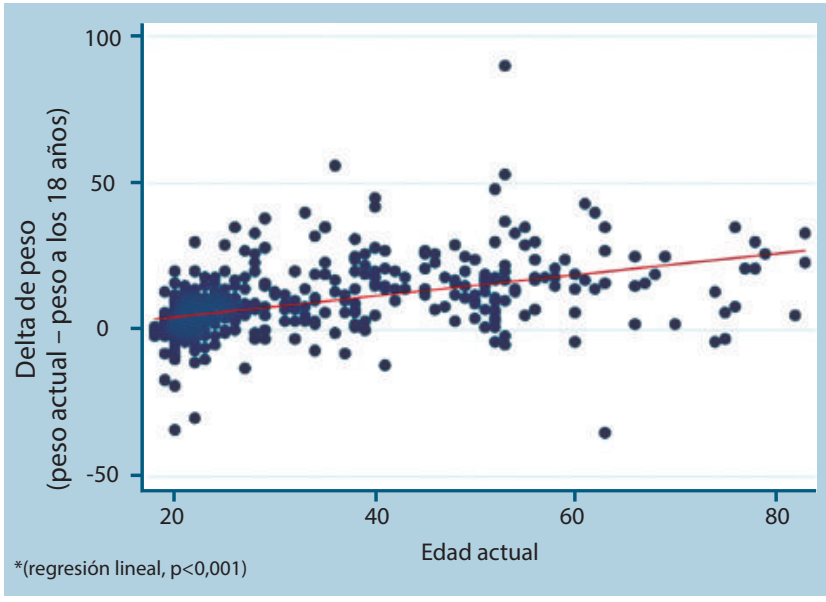

Se aplicó una regresión binomial robusta, donde fueron excluidas progresivamente las variables con la menor significancia estadística, obteniéndose un modelo donde sobresalen tres variables fuertemente relacionadas con tener exceso de peso (Tabla 3).

\section{Discusión}

En el presente estudio, los principales factores relacionados con tener exceso de peso en la edad adulta fueron la edad y el peso logrado a los 18 años. También se evidenció una ganancia de 0,35 kg por cada año cumplido. La detención del crecimiento longitudinal, el sedentarismo relacionado con la ocupación, la disminución de la tasa metabólica basal y los cambios hormonales propios de la edad son los factores más aceptados que soportan la ganancia de peso relacionada con el envejecimiento (20-22). Con mucha frecuencia se aprecia en la práctica clínica el anhelo constante de las personas de volver a tener el peso alcanzado a los 18 años; por ello, debemos aumentar la educación sobre este epifenómeno de la edad.

Por otro lado, el factor protector relacionado con la infancia más relevante para evitar el exceso de peso en nuestro estudio fue haber vivido en complejos residenciales; estos son espacios con amplias zonas sociales y recreativas seguras que permiten una mayor deambulación o prácticas deportivas. En este estudio, las personas que vivieron en estas urbanizaciones en la infancia presentaban 3,7 kg menos de peso frente a los que no. Existe evidencia que soporta una asociación entre los barrios amigables para caminar (walkability, en inglés) con menores tasas de exceso de peso en niños, adultos y ancianos (23-25). Un estudio realizado en Canadá que evaluó el índice de caminabilidad de 8777 barrios encontró menores prevalencias de sobrepeso, obesidad y diabetes mellitus en adultos entre los 30 y 64 años en los barrios con mayor caminabilidad (25). Este índice evaluó densidad poblacional, densidad residencial, acceso peatonal a destinos (por ejemplo, bancos, librerías, colegios) e interconectividad vial. En Colombia, si bien existe alguna información sobre caminabilidad en ciudades como Bogotá, no se ha evaluado la asociación con alguna patología metabólica (26).

Adicionalmente, se ha reportado en la literatura que personas que viven en zonas con altas tasas de inseguridad y criminalidad describen mayor riesgo de aumento en el IMC. Una revisión sistemática que incluyó 19 estudios encontró una asociación entre barrios con altas tasas de criminalidad con inactividad física y obesidad, principalmente en mujeres (27). Este tipo de variables no fueron indagadas en nuestro trabajo.

Aunque el Departamento Administrativo Nacional de Estadística (DANE) no reporta el tipo de vivienda discriminado por conjuntos cerrados o urbanizaciones similares, se infiere que estas son frecuentes en clase media y alta. En el presente estudio no se encontró relación entre el estrato socioeconómico a los 18 años con tener exceso de peso a esa edad ni en la actualidad. Se requiere una investigación con un mejor diseño metodológico que contemple una muestra estratificada por edad e ingresos económicos para profundizar el impacto del tipo de vivienda con el grado de actividad física y el exceso de peso.

Basado en lo anterior, las políticas de salud pública podrían ir encaminadas a promover los hábitos de vida saludable en el 
Tabla 1. Características demográficas y aspectos psicosociales en relación con tener actualmente exceso de eso $(n=490)$

\begin{tabular}{|c|c|c|}
\hline Variable & $\begin{array}{c}\text { Tener exceso } \\
\text { de peso } \\
\text { actualmente/n }\end{array}$ & $\begin{array}{l}\text { Valor } \\
\text { de } p^{*}\end{array}$ \\
\hline $\begin{array}{l}\text { Sexo } \\
\text { Mujer } \\
\text { Hombre }\end{array}$ & $\begin{array}{l}118 / 288(40,9 \%) \\
109 / 202(53,9 \%)\end{array}$ & 0,005 \\
\hline $\begin{array}{l}\text { Departamento - procedencia } \\
\text { Santander } \\
\text { Otro }\end{array}$ & $\begin{array}{l}170 / 386(44,0 \%) \\
57 / 104(54,8 \%)\end{array}$ & 0,05 \\
\hline $\begin{array}{l}\text { Actividad física en la } \\
\text { profesión } \\
\text { Leve } \\
\text { Moderado } \\
\text { Intenso }\end{array}$ & $\begin{array}{l}181 / 408(44,3 \%) \\
38 / 65(58,4 \%) \\
8 / 17(47,0 \%)\end{array}$ & 0,106 \\
\hline $\begin{array}{l}\text { Realiza actualmente algún } \\
\text { deporte } \\
\text { Sí } \\
\text { No }\end{array}$ & $\begin{array}{l}100 / 220(45,4 \%) \\
127 / 270(47,0 \%)\end{array}$ & 0,727 \\
\hline $\begin{array}{l}\text { Actividad física actual en el } \\
\text { padre } \\
\text { Leve } \\
\text { Moderada }\end{array}$ & $\begin{array}{l}151 / 301(50,1 \%) \\
76 / 189(40,2 \%)\end{array}$ & 0,031 \\
\hline $\begin{array}{l}\text { Actividad física actual en la } \\
\text { madre } \\
\text { Leve } \\
\text { Moderada }\end{array}$ & $\begin{array}{l}166 / 355(46,7 \%) \\
61 / 135(45,1 \%)\end{array}$ & 0,755 \\
\hline $\begin{array}{l}\text { Fumar con regularidad en } \\
\text { algún momento de la vida } \\
\text { Sí } \\
\text { No }\end{array}$ & $\begin{array}{l}153 / 366(41,8 \%) \\
74 / 124(59,6 \%)\end{array}$ & 0,001 \\
\hline $\begin{array}{l}\text { Consumo de sustancias } \\
\text { psicoactivas alguna vez en } \\
\text { la vida } \\
\text { Sí } \\
\text { No }\end{array}$ & $\begin{array}{l}38 / 84(45,2 \%) \\
189 / 406(46,5 \%)\end{array}$ & 0,826 \\
\hline $\begin{array}{l}\text { Hábito actual de alcohol } \\
\text { No consume } \\
\text { Social } \\
\text { Semanal } \\
\text { Diario }\end{array}$ & $\begin{array}{l}59 / 127(46,4 \%) \\
155 / 334(46,4 \%) \\
11 / 27(40,7 \%) \\
2 / 2(100 \%)\end{array}$ & 0,447 \\
\hline $\begin{array}{l}\text { Actividad física actual } \\
\text { IPAQ corto } \\
\text { Leve } \\
\text { Moderado } \\
\text { Intenso }\end{array}$ & $\begin{array}{l}60 / 137(43,8 \%) \\
84 / 177(47,4 \%) \\
83 / 175(47,4 \%)\end{array}$ & 0,768 \\
\hline
\end{tabular}

*Prueba de Chi cuadrado.
Tabla 2. Aspectos psicosociales de la infancia en relación con tener actualmente exceso de peso $(n=490)$

\begin{tabular}{|c|c|c|}
\hline Variable & $\begin{array}{l}\text { Tener exceso } \\
\text { de peso } \\
\text { actualmente/n }\end{array}$ & $\begin{array}{l}\text { Valor } \\
\text { de } p^{*}\end{array}$ \\
\hline $\begin{array}{l}\text { Edad gestacional } \\
\text { Pretérmino } \\
\text { Normal } \\
\text { Postérmino }\end{array}$ & $\begin{array}{l}27 / 63(42,8 \%) \\
173 / 375(46,1 \%) \\
11 / 17(64,7 \%)\end{array}$ & 0,270 \\
\hline $\begin{array}{l}\text { Peso al nacer } \\
\text { Bajo peso } \\
\text { Normal } \\
\text { Macrosomía }\end{array}$ & $\begin{array}{l}17 / 38(44,7 \%) \\
132 / 283(46,6 \%) \\
15 / 25(60 \%)\end{array}$ & 0,414 \\
\hline $\begin{array}{l}\text { Ser parte de una selección } \\
\text { deportiva en la adolescencia } \\
\text { Sí } \\
\text { No }\end{array}$ & $\begin{array}{l}110 / 255(43,1 \%) \\
117 / 235(49,7 \%)\end{array}$ & 0,140 \\
\hline $\begin{array}{l}\text { Alimentación en colegio } \\
\text { No comía } \\
\text { Lonchera } \\
\text { Dinero } \\
\text { Lonchera + dinero }\end{array}$ & $\begin{array}{l}36 / 56(64,2 \%) \\
40 / 100(40 \%) \\
87 / 177(49,1 \%) \\
64 / 157(40,7 \%)\end{array}$ & 0,010 \\
\hline $\begin{array}{l}\text { Consumo frecuente de } \\
\text { gaseosas en el colegio } \\
\text { Sí } \\
\text { No }\end{array}$ & $\begin{array}{l}145 / 291(49,8 \%) \\
82 / 199(41,2 \%)\end{array}$ & 0,06 \\
\hline $\begin{array}{l}\text { Víctima de matoneo en la } \\
\text { infancia } \\
\text { Sí } \\
\text { No }\end{array}$ & $\begin{array}{l}44 / 95(46,3 \%) \\
183 / 395(46,3 \%)\end{array}$ & 0,998 \\
\hline $\begin{array}{l}\text { Víctima de matoneo por el } \\
\text { peso en la infancia } \\
\text { Sí } \\
\text { No }\end{array}$ & $\begin{array}{l}23 / 42(54,7 \%) \\
204 / 448(45,5 \%)\end{array}$ & 0,252 \\
\hline $\begin{array}{l}\text { Maltrato verbal por los padres } \\
\text { Sí } \\
\text { No }\end{array}$ & $\begin{array}{l}26 / 60(43,3 \%) \\
201 / 430(46,7 \%)\end{array}$ & 0,620 \\
\hline $\begin{array}{l}\text { Maltrato físico por los padres } \\
\text { Sí } \\
\text { No }\end{array}$ & $\begin{array}{l}37 / 71(52,1 \%) \\
190 / 419(45,3 \%)\end{array}$ & 0,290 \\
\hline $\begin{array}{l}\text { Maltrato entre los padres } \\
\text { Sí } \\
\text { No }\end{array}$ & $\begin{array}{l}53 / 103(51,4 \%) \\
174 / 387(44,9 \%)\end{array}$ & 0,240 \\
\hline
\end{tabular}

*Prueba de Chi cuadrado. 
Tabla 3. Modelo de regresión binomial robusta. Factores demográficos y psicosociales asociados al exceso de peso en la actualidad (490 sujetos entrevistados)

\begin{tabular}{l|l|l}
\multicolumn{1}{c|}{ Variable } & OR (IC 95\%) & $\begin{array}{c}\text { Valor } \\
\text { de } \boldsymbol{p}\end{array}$ \\
\hline Edad actual & $1,11(1,04-1,19)$ & 0,002 \\
\hline $\begin{array}{l}\text { Edad de inicio de exceso de } \\
\text { peso }\end{array}$ & $0,96(0,90-1,04)$ & 0,398 \\
\hline $\begin{array}{l}\text { Peso a los 18 años } \\
\text { Vivir en la infancia en conjunto } \\
\text { residencial }\end{array}$ & $0,40(0,18-0,88)$ & $<0,001$ \\
\hline
\end{tabular}

IC: intervalo de confianza; OR: odds ratio.

niño, favoreciendo "ambientes saludables y seguros" para garantizar el peso más conveniente al iniciar su vida adulta.

Como debilidades del trabajo se identificaron el sesgo de recordación, ya que varias preguntas estaban relacionadas con la infancia, y el alcance limitado teniendo en cuenta que la encuesta se aplicó a una población de una institución o con ac- ceso a internet, así como el uso de un instrumento no validado para la ejecución del estudio.

\section{Conclusiones}

La edad, el peso a los 18 años y el no haber vivido en conjunto residencial en la infancia fueron los factores asociados más importantes con tener exceso de peso en la edad adulta en una población colombiana.

\section{Agradecimientos}

A Paola Andrea Torres Ortiz y Juan José Rodríguez, por su colaboración en la recolección de los datos.

\section{Contribución de los autores}

Todos los autores participaron en la preparación del proyecto, el diseño, la recolección de la muestra, el análisis de resultados y la redacción del manuscrito.

\section{Conflictos de interés}

Los autores declaran no tener conflictos de interés.

\section{Fuentes de financiación}

No se recibió financiación para la elaboración del estudio.

\section{Anexo 1. Factores demográficos y psicosociales asociados al exceso de peso en una población de Colombia}

Consentimiento informado:

La presente investigación es conducida por la Universidad Autónoma de Bucaramanga. La meta de este estudio es determinar la frecuencia y asociación de factores demográficos y psicosociales asociados a la población con sobrepeso y obesidad cuando se compara con población sin sobrepeso y obesidad.

Si accedes a participar en este estudio, se te pedirá completar una encuesta; esto tomará aproximadamente 10 minutos de tu tiempo. La participación en este estudio es estrictamente voluntaria. La información que se recoja será confidencial y no se usará para ningún otro propósito fuera de los de esta investigación. Tus respuestas al cuestionario serán codificadas usando un número de identificación y, por lo tanto, serán anónimas.

Para participar el único requisito es que tengas 18 años cumplidos o más y que vivas en Colombia. No podrás participar si estás en embarazo, si has tenido una enfermedad que haya provocado que pierdas al menos $10 \%$ de tu peso original y si te han hecho cirugías con el propósito de bajar de peso (cirugía bariátrica, manga gástrica, balón gástrico).

Si tienes alguna duda sobre este proyecto, puedes hacer preguntas en cualquier momento durante tu participación en él sin que eso te perjudique en ninguna forma. Si algunas de las preguntas durante la entrevista te parecen incómodas, tienes derecho de hacérselo saber al investigador o de retirarte del estudio tempranamente, si así lo decides.

Antes de finalizar la encuesta, revisa cuidadosamente haber respondido todas las preguntas correctamente.

Desde ya agradecemos tu participación.

He leído el consentimiento informado y acepto voluntariamente participar en la encuesta, reconociendo que la información será confidencial y no tiene otro fin por fuera del objetivo de la investigación. (ACEPTO / NO ACEPTO). 
1. ¿En qué departamento vives?

- Santander

- Cundinamarca

- Antioquia

- Norte de Santander

- Valle del Cauca

- Meta

- Arauca

- Otro

2. Sexo

- Masculino

- Femenino

3. Peso actual en kilogramos:

4. Talla en centímetros:

5. ¿Consideras que te encuentras en exceso? (SÍ / NO)

a. Si tu respuesta es "SÍ" contesta la pregunta número 6, si tu respuesta es "NO", por favor pasa a la pregunta 7.

6. ¿Desde qué edad consideras que tienes exceso de peso?

7. De las siguientes imágenes, ¿cuál consideras que refleja mejor tu silueta actual?
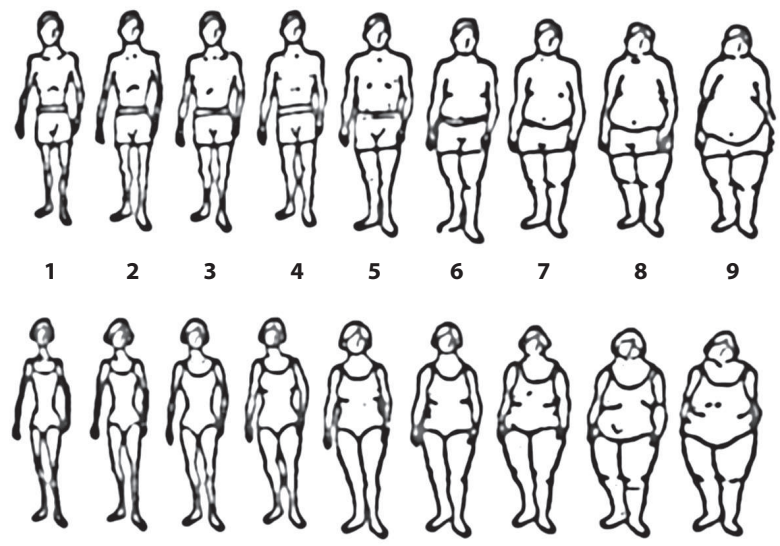

8. Edad en años cumplidos:

9. ¿En qué lugar permaneciste la mayor parte del tiempo en tu infancia? (RURAL / URBANO)

10. ¿En qué tipo de vivienda permaneciste la mayor parte del tiempo en tu infancia? (CASA / APARTAMENTO)

11. Durante tu infancia, ¿viviste en conjunto cerrado? (SÍ / NO)

12. Residencia actual (RURAL / URBANO)

13. ¿Cuál fue tu máximo nivel educativo alcanzado?

- Básica primaria

- Básica secundaria

- Secundaria media vocacional

- Técnico o tecnólogo

- Profesional

- Posgrado

14. Selecciona el grado de actividad física que consideras que involucra el desarrollo de tu ocupación

- Leve (por ejemplo: estudiante, oficina, docente, conductor)
- Moderado (por ejemplo: servicios generales, mensajería)

- Intenso (por ejemplo: agricultor, ganadero, constructor)

15. Edad gestacional al nacimiento

- Prematuro (hasta 37 semanas u 8 meses de embarazo)

- A término (más de 38 semanas o 9 meses de embarazo)

- Post término (más de 41 semanas)

- No sabe

16. Peso al nacer

- Menor a $2500 \mathrm{~g}$ (bajo peso)

- 2500gr a $4000 \mathrm{~g}$ (peso normal)

- Mayor a 4000 g (macrosomía o grande para la edad gestacional)

17. Meses de lactancia materna recibidos:

18. Número de hermanos:

19. Enfermedades padecidas en la infancia

- Asma

- Epilepsia

- Nefropatías

- Cardiopatías

- Otras enfermedades neurológicas

- Obesidad

- Otras enfermedades endocrinológicas (hipotiroidismo, diabetes, síndrome de Cushing, etc.)

- Ninguna de las anteriores

- Otra

20. Medicamentos recibidos en la vida durante 3 meses o más

- Antidiabéticos orales

- Insulinas

- Anticonvulsivantes

- Antidepresivos

- Antipsicóticos

- Corticoides

- Ninguno de los anteriores

- Otro

21. ¿Quién fue tu cuidador principal la mayor parte del tiempo en tu infancia?

- Madre

- Padre

- Madre y padre

- Abuelos

- Otro miembro de la familia

- Niñera

22. Estrato socioeconómico antes de los 18 años $(0$ / 1 / 2 / 3 / 4 / 5 / 6)

23. Selecciona el grado de actividad física que consideras el desarrollo de la ocupación de tu padre

- Leve (por ejemplo: estudiante, oficina, docente, conductor)

- Moderado (por ejemplo: servicios generales, mensajería)

- Intenso (por ejemplo: agricultor, ganadero, constructor)

24. Selecciona el grado de actividad física que consideras que involucra el desarrollo de la ocupación de tu madre: 
- Leve (por ejemplo: estudiante, oficina, docente, conductor)

- Moderado (por ejemplo: servicios generales, mensajería)

- Intenso (por ejemplo: agricultor, ganadero, constructor)

25. ¿Tu padre realiza con frecuencia algún tipo de actividad física? (SÍ / NO)

26. ¿Tu madre realiza con frecuencia algún tipo de actividad física? (Sí / NO)

27. ¿Tu madre consume con frecuencia bebidas alcohólicas? (Sí / NO)

28. ¿Tu padre consume con frecuencia bebidas alcohólicas? (Sí / NO)

29. ¿Perteneciste a alguna selección deportiva en la infancia o adolescencia? (SÍ / NO)

30. ¿Cuánto tiempo (en años) practicaste deporte en tu infancia $o$ adolescencia?

31. Señala las décadas de tu vida dentro de las cuáles practicaste algún tipo de actividad física

- $0-10$ años

- 10-20 años

- 20-30 años

- 30-40 años

- 40-50 años

- 50-60 años

- 60-70 años

- 70-80 años

- 80-90 años

32. ¿Cuál era tu peso en kilogramos a los 18 años?:

33. Actualmente, ¿realizas ejercicio o practicas algún deporte? (SÍ / NO)

Piensa en todas las actividades intensas que realizaste en los últimos 7 días. Las actividades físicas intensas se refieren a aquellas que implican un esfuerzo físico intenso y que lo hacen respirar mucho más intensamente que lo normal. Piensa solo en aquellas actividades físicas que realizaste durante por lo menos 10 minutos seguidos.

34. Durante los últimos 7 días, ¿en cuántos realizaste actividades físicas intensas tales como levantar pesos pesados, cavar, hacer ejercicios aeróbicos o andar rápido en bicicleta? (Si la respuesta es 0 salta a la pregunta 36 )

35. Habitualmente, $i$ cuánto tiempo en total (en minutos) dedicaste a una actividad física intensa en uno de esos días?

Piensa en todas las actividades moderadas que tu realizaste en los últimos 7 días. Las actividades moderadas son aquellas que requieren un esfuerzo físico moderado que lo hace respirar algo más intensamente que lo normal. Piensa solo en aquellas actividades físicas que realizaste durante por lo menos 10 minutos seguidos.

36. Durante los últimos 7 días, ¿en cuántos días hiciste actividades físicas moderadas como transportar pesos livia- nos, andar en bicicleta a velocidad regular o jugar dobles de tenis? No incluyas caminar. (Si tu respuesta es 0 ve a la pregunta 38)

37. Habitualmente, ¿cuánto tiempo en total (en minutos) dedicaste a una actividad física moderada en uno de esos días? Piensa en el tiempo que dedicaste a caminar en los últimos 7 días. Esto incluye caminar en el trabajo o en la casa, para trasladarse de un lugar a otro, o cualquier otra caminata que tu podrías hacer solamente para la recreación, el deporte, el ejercicio o el ocio.

38. Durante los últimos 7 días, ¿en cuántos caminaste por lo menos 10 minutos?

39. Habitualmente, ¿cuánto tiempo en total (en minutos) dedicaste a caminar en uno de esos días?

La última pregunta es acerca del tiempo que pasaste sentado durante los días hábiles de los últimos 7 días. Esto incluye el tiempo dedicado al trabajo, en la casa, en una clase, y durante el tiempo libre. Puede incluir el tiempo que pasaste sentado ante un escritorio, visitando amigos, leyendo, viajando en bus, o sentado o recostado mirando la televisión.

40. Durante los últimos 7 días, ¿cuánto tiempo (en horas) pasaste sentado durante un día hábil?

41. Alimentación en el colegio

- Dinero

- Lonchera

- Ambos

42. ¿Con qué frecuencia consumías gaseosas o jugos de caja en el colegio? (SÍ / NO)

43. ¿Fuiste víctima de bullying o matoneo en el colegio? (SÍ / NO)

44. ¿Fuiste víctima de bullying o matoneo por el peso en el colegio? (SÍ / NO)

45. ¿Recibiste maltrato verbal por tus padres? (Sí / NO)

46. ¿Recibiste maltrato físico por tus padres? (SÍ / NO)

47. ¿Hubo maltrato físico o verbal entre tus padres? (Sí / NO)

48. Plan familiar de preferencia los domingos hasta los 18 años

- Ir a misa u otro culto religioso

- Paseo familiar

- Almuerzo familiar

- Juego, deporte, ciclovía

- Permanecer en casa

49. ¿Has fumado cigarrillo con regularidad en algún momento de tu vida? (SÍ / NO)

a. Si la respuesta es "SÍ" responde las preguntas 50 y 51. Si tu respuesta es "NO" salta a la pregunta 52

50. ¿Cuántos cigarrillos al día fumas o fumabas?

51. ¿Por cuánto tiempo (en años) has fumado cigarrillo?

52. ¿Has consumido sustancias psicoactivas? (Sí / NO)

53. ¿Con qué frecuencia consumes alcohol?

- Consumidor social 
- Consumidor semanal

- Consumidor diario

- No consume

54. ¿Alguna vez has ido al psicólogo o al psiquiatra?

55. ¿Cuántos días a la semana consumes frutas?

56. ¿Cuántos días a la semana consumes verduras?

57. ¿Cuántos días a la semana consumes jugos de fruta?

58. ¿Cuántos días a la semana consumes sopas o caldos?

59. ¿Cuántos días a la semana consumes gaseosas o jugos de caja?

60. ¿Te mencionaban tus padres frases como: "NO TE LEVANTAS DE LA MESA HASTA QUE TERMINES DE COMER"? (SÍ / NO)
61. ¿Te mencionaban tus padres frases como "DEBES COMERTE TODO PORQUE HAY NIÑOS QUE NO TIENEN NADA PARA COMER"?

62. ¿Te mencionaban tus padres frases como: "LA COMIDA NO SE PUEDE BOTAR"?

63. Durante tu infancia, ¿con qué te premiaban tus padres?

- Juguetes

- Dulces

- Paseos

- Libros

- TVy videojuegos

- Ninguno de los anteriores

\section{Referencias}

1. Ng M, Fleming T, Robinson M, Thomson B, Graetz N, Margono C, et al. Global, regional, and national prevalence of overweight and obesity in children and adults during 1980-2013: a systematic analysis for the Global Burden of Disease Study 2013. Lancet. 2014;384(9945):766-81. doi: 10.1016/ S0140-6736(14)60460-8

2. Ministerio de Salud y Protección Social, Instituto Nacional de Salud, Instituto de Prosperidad Social, Instituto Colombiano de Bienestar Familiar, Universidad Nacional de Colombia. Encuesta Nacional de Situación Nutricional de Colombia (ENSIN). 2015:448-463. Disponible en: https://www. minsalud.gov.co/sites/rid/Lists/BibliotecaDigital/RIDE/VS/ED/GCFI/ libro-ensin-2015.pdf

3. Schwartz MW, Seeley RJ, Zeltser LM, Drewnowski A, Ravussin E, Redman LM, et al. Obesity Pathogenesis: An Endocrine Society Scientific Statement. Endocr Rev. 2017;38(4):267-96. doi: 10.1210/er.2017-00111

4. Kushner RF. Clinical assessment and management of adult obesity. Circulation. 2012;126(24):2870-7. doi: 10.1161/CIRCULATIONAHA.111.075424

5. Preventing and Managing the Global Epidemic. Génova, Suiza: World Health Organization Obesity; 1998. Disponible en: https://bit.ly/2Wi2z4a

6. Swinburn BA, Caterson I, Seidell JC, James WP. Diet, nutrition and the prevention of excess weight gain and obesity. Public Health Nutr. 2004;7(1A):123-46. doi: 10.1079/phn2003585

7. Álvarez-Castaño LS, Goez-Rueda JD, Carreño-Aguirre C. Factores sociales y económicos asociados a la obesidad: los efectos de la inequidad y de la pobreza. Revista Gerencia y Políticas de Salud. 2012;11(23):98-110. Disponible en: https://bit.ly/37pgvzv

8. Herrera BM, Lindgren CM. The genetics of obesity. Curr Diab Rep. 2010;10(6):498-505. doi: 10.1007/s11892-010-0153-z

9. Truett J, Cornfield J, Kannel W. A multivariate analysis of the risk of coronary heart disease in Framingham. J Chronic Dis. 1967;20(7):511-24. doi: 10.1016/0021-9681(67)90082-3

10. Ganz ML, Wintfeld N, Qian L, Alas V, Langer J, Hammer M. The association of body mass index with the risk of type 2 diabetes: a case control study nested in an electronic health records system in the United States. Diabetol Metab Syndr. 2014;6(1):50. doi: 10.1186/1758-5996-6-50

11. Pico SM, Bergonzoli G, Contreras A. Risk factors associated with the metabolic syndrome in Cali, Colombia (2013): A case-control study. Biomedica. 2019;39(1):46-54. doi: 10.7705/biomedica.v39i1.3935

12. Begum GS, Shariff A, Ayman G, Mohammad B, Housam R, Khaled N. Assessment of risk factors for development of polycystic ovarian syndrome. Int J Contemp Res. 2017;4(1):77-83. Disponible en: https://bit.ly/3nu0nCG

13. Lu F, Hu E, Xu L, Chen L, Wu J, Li H, et al. The relationship between obesity and the severity of non-alcoholic fatty liver disease: systematic review and meta-analysis. Expert Rev Gastroenterol Hepatol. 2018;12(5):491-502. doi: 10.1080/17474124.2018.1460202

14. Hein M, Lanquart JP, Loas G, Hubain P, Linkowski P. Prevalence and risk factors of moderate to severe obstructive sleep apnea syndrome in insom- nia sufferers: a study on 1311 subjects. Respir Res. 2017;18(1):135. doi: 10.1186/s12931-017-0616-8

15. Zheng $\mathrm{H}$, Chen $\mathrm{C}$. Body mass index and risk of knee osteoarthritis: systematic review and metanalysis of prospective studies. BMJ Open. 2015;5(12):e007568. doi: 10.1136/bmjopen-2014-007568

16. Aune D, Navarro-Rosenblatt DA, Chan DS, Vingeliene S, Abar L, Vieira AR, et al. Anthropometric factors and endometrial cancer risk: a systematic review and dose-response meta-analysis of prospective studies. Ann Oncol. 2015;26(8):1635-48. doi: 10.1093/annonc/mdv142

17. Bardou M, Barkun AN, Martel M. Obesity and colorectal cancer. GUT. 2013;62(6):933-47. doi: 10.1136/gutjnl-2013-304701

18. Rohde K, Keller M, la Cour Poulsen L, Blüher M, Kovacs P, Böttcher Y. Genetics and epigenetics in obesity. Metabolism. 2019;92:37-50. doi: 10.1016/j. metabol.2018.10.007

19. González-Muniesa P, Mártinez-González MA, Hu FB, Després JP, Matsuzawa Y, Loos RJF, et al. Obesity. Nat Rev Dis Primers. 2017;3:17034. doi: 10.1038/nrdp.2017.34

20. Pérez LM, Pareja-Galeano H, Sanchis-Gomar F, Emanuele E, Lucia A, Gálvez BG. 'Adipaging': ageing and obesity share biological hallmarks related to a dysfunctional adipose tissue. J Physiol. 2016;594(12):3187-207. doi: 10.1113/JP271691

21. Solomon TP, Marchetti CM, Krishnan RK, Gonzalez F, Kirwan JP. Effects of aging on basal fat oxidation in obese humans. Metabolism. 2008;57(8):1141-7. doi: 10.1016/j.metabol.2008.03.021

22. Davis S, Castelo-Branco C, Chedraui P, Lumsden M, Nappi R, Shah D, et al Understanding weight gain at menopause. Climacteric. 2012;15(5):41929. doi: $10.3109 / 13697137.2012 .707385$

23. Duncan DT, Sharifi M, Melly SJ, Marshall R, Sequist TD, Rifas-Shiman SL, et al. Characteristics of walkable built environments and BMI z-scores in children: evidence from a large electronic health record database. Environ Health Perspect. 2014;122(12):1359-65. doi: 10.1289/ehp.1307704

24. Creatore MI, Glazier RH, Moineddin R, Fazli GS, Johns A, Gozdyra P, et al Association of Neighborhood Walkability With Change in Overweight, Obesity, and Diabetes. JAMA. 2016;315(20):2211-20. doi: 10.1001/ jama.2016.5898

25. Michael YL, Nagel CL, Gold R, Hillier TA. Does change in the neighborhood environment prevent obesity in older women? Soc Sci Med. 2014;102:129 37. doi: 10.1016/j.socscimed.2013.11.047

26. Gutiérrez-López JA, Caballero-Pérez YB, Escamilla-Triana RA. Índice de caminabilidad para la ciudad de Bogotá. Revista de Arquitectura. 2019;21(1):8-20. doi: 10.14718/RevArq.2019.21.1.1884

27. Yu E, Lippert AM. Neighborhood crime rate, weight-related behaviors, and obesity: A systematic review of the literature. Sociol Comp. 2016;10(3):187-207. doi: $10.1111 /$ soc4.12356 
Abbott

$$
\text { Guacerna }
$$

Joma el control de tu diabetes*

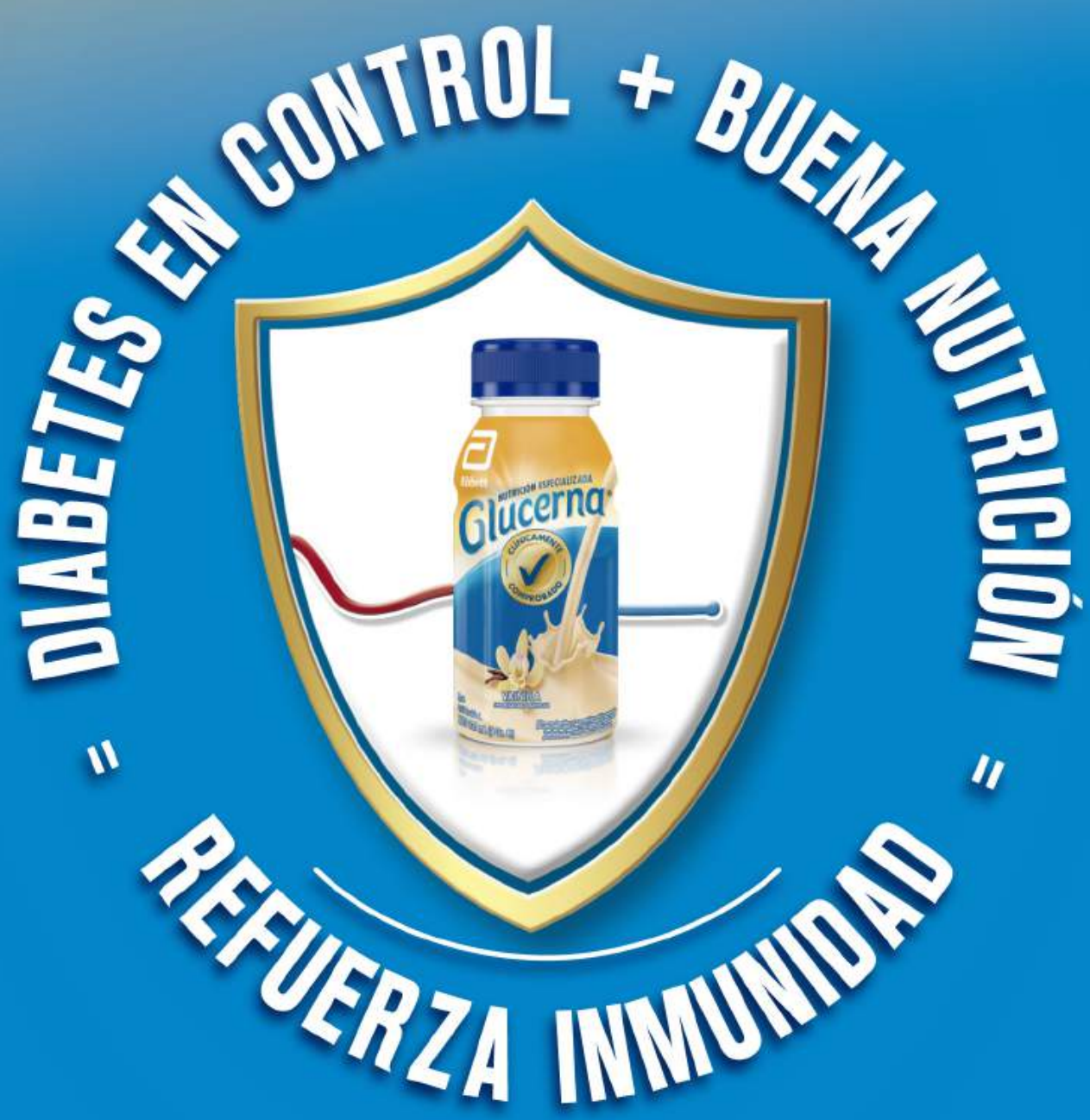

Glucerna $a^{\oplus}$ Alimento para propósitos médicos especiales, Glucerna ${ }^{\circledR}$ Líquida. Registro Sanitario:

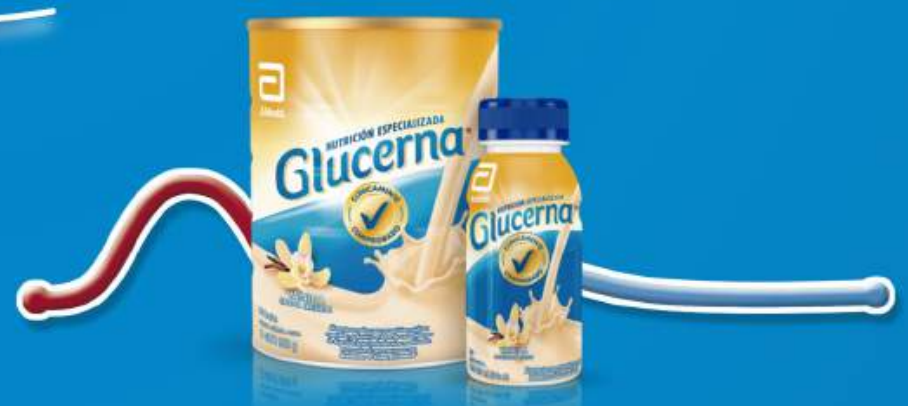

